\title{
Magnetoencephalography as a Putative Biomarker for Alzheimer's Disease
}

\author{
Edward Zamrini, ${ }^{1}$ Fernando Maestu, ${ }^{2}$ Eero Pekkonen, ${ }^{3}$ Michael Funke, ${ }^{1}$ Jyrki Makela, \\ Myles Riley, ${ }^{1}$ Ricardo Bajo, ${ }^{2}$ Gustavo Sudre, ${ }^{5}$ Alberto Fernandez, ${ }^{2}$ Nazareth Castellanos, ${ }^{2}$ \\ Francisco del Pozo, ${ }^{2}$ C. J. Stam, ${ }^{6}$ Bob W. van Dijk, ${ }^{7}$ Anto Bagic, ${ }^{8}$ and James T. Becker ${ }^{8,9,10,11}$
}

\author{
${ }^{1}$ Department of Neurology, University of Utah, Salt Lake City, UT 84112, USA \\ ${ }^{2}$ Laboratory of Cognitive and Computational Neuroscience, Center for Biomedical Technology, \\ Complutense University of Madrid and Technical University of Madrid, Madrid 28040, Spain \\ ${ }^{3}$ Department of Neurology, University of Helsinki, Helsinki FI-00029, Finland \\ ${ }^{4}$ BioMag Laboratory, HUSLAB, Helsinki University Central Hospital, Helsinki FI-00029, Finland \\ ${ }^{5}$ Center for the Neural Basis of Cognition, Carnegie-Mellon University, Pittsburgh, PA15213, USA \\ ${ }^{6}$ Department of Clinical Neurophysiology, VU University Medical Center, 1007 MB Amsterdam, The Netherlands \\ ${ }^{7}$ Physics and Medical Technology Center, VU University Medical Center, 1007 MB Amsterdam, The Netherlands \\ ${ }^{8}$ Department of Neurology, University of Pittsburgh, Pittsburgh, PA 15213-3206, USA \\ ${ }^{9}$ Department of Psychiatry, University of Pittsburgh, Pittsburgh, PA 15213-3206, USA \\ ${ }^{10}$ Department of Psychology, University of Pittsburgh, Pittsburgh, PA 15250, USA \\ ${ }^{11}$ Neuropsychology Research Program, University of Pittsburgh, Suite 830, 3501 Forbes Avenue, Pittsburgh, PA 15213, USA
}

Correspondence should be addressed to James T. Becker, beckerjt@upmc.edu

Received 1 November 2010; Accepted 15 February 2011

Academic Editor: James B. Brewer

Copyright (c) 2011 Edward Zamrini et al. This is an open access article distributed under the Creative Commons Attribution License, which permits unrestricted use, distribution, and reproduction in any medium, provided the original work is properly cited.

\begin{abstract}
Alzheimer's Disease (AD) is the most common dementia in the elderly and is estimated to affect tens of millions of people worldwide. AD is believed to have a prodromal stage lasting ten or more years. While amyloid deposits, tau filaments, and loss of brain cells are characteristics of the disease, the loss of dendritic spines and of synapses predate such changes. Popular preclinical detection strategies mainly involve cerebrospinal fluid biomarkers, magnetic resonance imaging, metabolic PET scans, and amyloid imaging. One strategy missing from this list involves neurophysiological measures, which might be more sensitive to detect alterations in brain function. The Magnetoencephalography International Consortium of Alzheimer's Disease arose out of the need to advance the use of Magnetoencephalography (MEG), as a tool in AD and pre-AD research. This paper presents a framework for using MEG in dementia research, and for short-term research priorities.
\end{abstract}

\section{Introduction}

Alzheimer's disease $(\mathrm{AD})$ is the major cause of clinical dementia in the elderly. As many as 35.6 million people worldwide may currently be living with dementia, with the prevalence increasing to 65.7 million by 2030 and 115.4 million by 2050; two thirds of these people will likely have AD (http://www.alz.co.uk/research/files/WorldAlzheimerReport -ExecutiveSummary.pdf). The incidence and prevalence of $\mathrm{AD}$ begins to rise as individuals reach the age of 65 so that by the time they are in their $80 \mathrm{~s}$ and $90 \mathrm{~s}$, the risk of clinical dementia is nearly $50 \%$. However, in spite of the fact that the risk of the clinical syndrome, Alzheimer's dementia, is greatest in the later years of life, the pathological processes, Alzheimer's Disease, begin 10-20 or more years before clinical onset. This means that treatment strategies aimed at disease modification will be most efficacious if they can occur during the period when the pathological changes are occurring, but have not yet exhibited themselves as clinical signs and symptoms. 
Although the diagnostic criteria for AD have been well codified since the early 1980s [1-3], there has been a recent upsurge in interest in studying individuals who are in the transitional stage between normal cognition and full-blown dementia. This syndrome, referred to as Mild Cognitive Impairment (MCI) [4-9], has been the focus of intense study and there are many who believe that in the absence of other medical comorbidities, individuals with MCI, in fact, have clinical AD in its earliest stages [10-13].

At the same time, with the development of new biological technologies, there is an increased interest in adding biomarkers as a form of pathological confirmation of the clinical diagnosis of $\mathrm{AD}$. The current research diagnostic standard-those of the NINCDS/ADRDA [1] gives a probabilistic estimate of the risk of pathological AD in the context of a clinical dementia syndrome. The final diagnosis of "Definite" AD can only be made in the presence of a sufficient number of senile plaques and neurofibrillary tangles, that is, a neuropathological examination is required [14-16]. In life, however, clinical diagnosis is rated in terms of a set of clear criteria that focus not only on core signs and symptoms, but also on the presence or absence of comorbid factors that could, in and of themselves, cause a dementia syndrome. In the absence of such comorbid conditions, an individual is thought to have "Probable" AD. When such conditions are present, then the certainty of diagnosis is reduced to the level of "Possible".

Recent attempts at reformulating the diagnostic criteria have focused on the potential utility of biomarkers including brain structural and functional imaging, cerebrospinal fluid measures of amyloid beta, and alterations in brain chemistry measured using positron emission tomography (PET) [17] (cf. [18]). Some biomarkers have shown excellent predictive validity relative to neuropathology including MRI measures of brain structural integrity, PET and measures of cerebral blood flow and metabolism. However, "(w)hether one of these measures or a combination of them is more sensitive than the other, and whether quantitative values provide more information than a dichotomous rating are yet to be determined conclusively" (http://www.alz.org/research/ diagnostic_criteria/, accessed 09 Sep, 2010).

Biomarkers measured at the time of the diagnosis of $\mathrm{AD}$ are excellent in predicting the presence of pathology at the time of autopsy, 8-10 years later. However, they are less efficient at detecting the presence of pathological change prior to the onset of clinical symptoms (cf., $[19,20])$. This could be due, in part, to the mechanisms of the initiation of the molecular cascade leading to amyloid deposition [21]. While PET imaging is particularly useful for measuring brain metabolism, alterations in amyloid deposits, and in neurochemistry, the temporal and spatial resolution of a PET scanner is such that it reduces its sensitivity to the very early and statistically small changes in brain function (See Figure 1). MRI has improved temporal and spatial resolution relative to PET, but we likely need higher temporal resolution than is afforded by MR techniques in order to identify the earliest functional changes of disease in the preclinical stage. Thus, it seems necessary to have a biomarker that (1) measures neuronal activity directly, (2) has good temporal and spatial

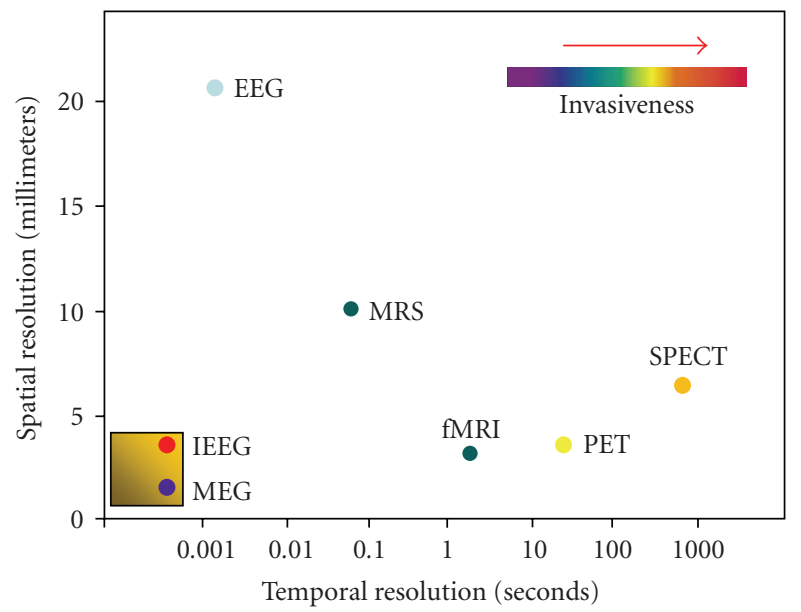

FIgURE 1: Graphic showing the relative spatial and temporal resolutions of common neuroimaging techniques (EEG: Electroencephalography, IEEG: Invasive Electroencephalography, MEG: Magnetoencephalography, MRS: Magnetic Resonance Spectroscopy, fMRI: functional MRI, SPECT: Single Photon Emission Cranial Tomography, and PET: Positron Emission Tomography).

resolution, and (3) is able to evaluate functional networks and the associated neuronal code (i.e., oscillatory activity).

One potential biomarker that has received relatively little attention is that of electrophysiological/biomagnetic changes in the brain. These measures are potentially very useful because although their spatial resolution is similar to that of MRI, their temporal resolution is as many as three orders of magnitude better than other existing functional tools. Furthermore, these techniques are noninvasive, measure neuronal activity directly, and provide valuable information regarding the frequency of oscillatory activity. Consequently, these measures may provide the best index of the earliest functional changes that may occur secondary to neuropathological processes, but prior to the onset of the clinical dementia syndrome. In order to be most effective, however, these markers must have predictive validity relative to the neuropathology of $\mathrm{AD}$, as well as modest intraand intersubject variability. And, they must be stable over reasonable time course (i.e., 3-6 months) in order to be useful to track change in disease, or response to medication.

The application of electroencephalography (EEG) in the clinical evaluation of $\mathrm{AD}$ patients has a long tradition $[22,23]$, and it is now generally accepted that the EEG in mild $\mathrm{AD}$ is marked by an increase in theta activity, accompanied by a decrease in beta and alpha activity [24]; more severe AD is marked by an increase in delta power [25]. This pattern of results is referred to as the "slowing" of the AD patients" EEG [26, 27]. Further, it appears that differences in EEG abnormalities based on visual ratings may be associated with different cognitive profiles [28]. AD patients with normal EEGs show the best performance in cognitive tasks, and only a moderate memory disturbance. Those patients with focal abnormalities (sharp waves or focal slow-wave activity) had average performance on the cognitive tasks, with a moderate alteration in semantic verbal fluency and poor performance 
on the Trail-Making B test. Patients with diffuse slowing only (a dominant frequency below $8 \mathrm{~Hz}$ ) showed the worst performance in all tasks. Finally, AD patients with both focal and diffuse EEG abnormalities group showed good performance in episodic memory tasks and a significant deterioration in working memory and executive functions.

EEG has also been investigated in MCI patients, and a similar pattern of slowing was found. Theta band power and coherence differs significantly between MCI patients and healthy subjects [29], and alpha and theta relative power in the left temporo-occipital derivation correctly classified $85 \%$ of MCI subjects who would later develop AD [30]. A more anterior localization of theta and alpha activity is also a good predictor of future development of $\mathrm{AD}$ from MCI [31]. Generally, however, MCI subjects tend to show intermediate EEG parameters between those of ADs and controls. There is a slowing of the spectral profile in GDS stage 3 (i.e., MCI subjects), as compared to controls [32]. Patients classified as GDS 4 or 5 (i.e. clinical dementia) have very similar profiles to MCI patients; only patients with moderate to severe dementia had a frequency pattern that was distinct from all other patients groups. Similarly, increases in theta and reductions in alpha power can distinguish $\mathrm{AD}$ patients and controls, but cannot distinguish between MCIs and controls [33]. MCI subjects' EEG parameters are not only intermediate between those of controls and $\mathrm{AD}$, but also show a considerable overlap [27]. Babiloni and colleagues [34] have suggested that traditional EEG power spectrum and amplitude analysis might not be sensitive enough to differentiate MCI patients from healthy control subjects.

Another method of analysis, estimating coherence/synchronization, is an example of a technique that could improve classification of MCI. Coherence analysis is widely used and represents a normalized linear measure of the correlations between two signals as a function of frequency $[35,36]$. There is a decrease in coherence values in alpha and beta bands in $\mathrm{AD}$ patients (for a review, see [27]). In the case of MCI, there is a decrease of intrahemispheric frontoparietal EEG coherence and an increase of temporal interhemispheric coherence compared to elderly controls. [37]. Synchronization likelihood (SL) [38] has been used to study the brain activity in MCI, and was found to be significantly decreased in the $14-18$ and $18-22 \mathrm{~Hz}$ bands in $\mathrm{AD}$ patients compared with both $\mathrm{MCI}$ subjects and healthy controls [39]. Unfortunately, SL was similar in the controls and MCI patients.

Most of resting state EEG studies in MCI show a pattern of increased low-frequency activity accompanied by a decrease of coherence or synchronization. By contrast, activation studies tend to show increased SL. In a study of MCI patients at rest and performing a visual working memory task, significant differences between the patients and controls were found only in the alpha- 2 band during the working memory condition (i.e., not at rest) [40]. Indeed, there is an increase in SL in MCI during the performance of a working memory task, and this increase was associated with the risk for the progression to $\mathrm{AD}$ [41]. These paradoxical findings may be related to a compensatory mechanism in the brain during the first stages of cognitive deterioration.
Although coherence and SL are probably two of the most broadly used estimates of EEG connectivity in $\mathrm{AD}$, a variety of synchronization estimates are being used. For example, Kramer and colleagues [42] compared phase synchrony and two measures of nonlinear interdependency in $\mathrm{AD}$ patients, MCI subjects, and healthy controls. Only phase synchrony and one of the nonlinear estimates allowed the discrimination of $\mathrm{AD}$ patients from controls, and $\mathrm{AD}$ and $\mathrm{MCI}$ subjects. None of the measures was capable of discriminating the MCI patients from the controls. Dauwels and collaborators [43] tested as many as 20 synchrony measures, on five minutes of resting EEG data in $25 \mathrm{MCI}$ patients and 56 healthy controls. Overall, the MCI patients had lower synchronization values compared to controls, but only two were significant after correction for multiple comparisons. Those estimates were the so-called "Full frequency directed transfer function", and the $\rho$ parameter of the Stochastic event synchrony. Although their pattern of results may be due to a disconnection syndrome in the AD patients, Dauwels and coworkers [43] highlighted the fact that their results could not be due to a disconnection syndrome alone (e.g., [44]); the AD patients showed not only a reduced synchrony but also an increase of asynchronous activity.

Thus, while EEG provides important information about $\mathrm{AD}$, it does not appear adequate to detect preclinical disease. In this paper we will focus on the potential utility of magnetoencephalography (MEG) to detect functional changes in the brain secondary to AD pathology, prior to symptom onset. MEG is a sensitive tool for measuring magnetic fields that correspond to electrical currents in the brain, and that has been extremely useful for non-invasive studies of epilepsy. MEG technology was born 40 years ago [45] and has been used for more than 20 years within multimodal neuroimaging, but only relatively recently has it begun to be used more extensively in clinical and research settings.

While it is true that MEG it is not a widespread technique, the number of MEG centers has increased dramatically in Europe and in America over the last ten years. In the past, the analysis of MEG data was difficult and required very specialized methods. More recently, equipment manufacturers have developed new software that is relatively userfriendly allowing physicians to analyze MEG data; there is new freeware (EEGlab; Brain Storm; SPM8, FieldTrip), and commercial products (Curry, BESA) are available, as well. It is still true that for advanced analysis such as connectivity or network analysis a high degree of analytic specialization is necessary, but this is a normal stage in the development of any newer technology.

MEG records activity in the brain based on the magnetic fields induced by synchronized neuronal currents [46, 47] and can monitor the activation of synchronously firing neuronal populations with a submillisecond temporal resolution [46-48]. However, since the magnetic signals of the brain vary between $10^{2}$ (evoked cortical activity) and $10^{3}$ femtoteslas (fT) (the human alpha rhythm), and the magnetic field of the earth is in the neighborhood of $10^{9} \mathrm{fT}$, the method has to balance two problems: the weakness of the signal and the strength of the noise [47]. These issues are addressed by using hundreds of extremely sensitive 
superconducting quantum interference devices incorporated in the whole head system (for recording simultaneously from the entire brain) and used in a magnetically shielded room [47].

MEG has several advantages over other measures of electrical activity of the brain including its freedom from the requirement for a reference electrode which has the potential to improve calculations of the electrical power and source localization of the signal. Because there is no need for a reference point in MEG studies, this facilitates synchronization and coherence analyses. Connectivity measures and source reconstruction solutions depend to some extent on the positioning of the reference channels, thus the lack of a reference gives MEG an advantage over EEG. MEG uses many more detectors than traditional EEG sensor arrays in many machines, and this can obtain whole head coverage of signal very rapidly. Because magnetic fields are more transparent through biological tissue, high-frequency bands have better signal-to-noise ratios than EEG where electrical currents are affected by the resistance of biological tissues and distorted by the skull. This allows for better spatial resolution and thus better localization of the sources of electrical activity from MEG compared to EEG. The resistance of the biological tissues to electrical current also produces severe attenuation of the EEG signal in the case of the high-gamma band. MEG, on the other hand, is much more sensitive to this range of frequencies. In the last decade there is increasing evidence of the close relation between the gamma band and cognition.

MEG has several potential advantages over functional MRI (fMRI), another potential biomarker for AD. First, it has a much greater temporal resolution, and as one of the consequences of neurodegeneration is slowed mentation, this may mean that MEG is better able to measure such subtle delays in local and regional responses. Second, by and large, metallic fragments in the body are not contraindications for the scan, although if they are large, or are too close to the head they render the MEG signals too noisy. Having a 306-sensor, whole head system may allow for easier comprehensive spatial sampling and thus better suppression of interference, and advanced processing tools can reduce unwanted interference [49]. Third, and most important, is the fact that MEG does not rely on the hemodynamic response (see [50], for discussion). fMRI measures neuronal activity only indirectly. When a group of neurons become activated there is a local increase (about $4000 \mathrm{~ms}$ after the activation) in blood flow which makes it impossible to measure oscillatory activity in the most relevant frequency bands. It is the analysis of oscillatory activity afforded by MEG that allows for the estimation of phase synchronization indices between brain regions. Intracellular currents in large neuronal assemblies of at least $\sim 10^{4-5}$ pyramidal cells in parallel orientation can be measured more directly, providing "a more direct index of sensory, motor, and cognitive taskspecific activation compared with methods that rely on hemodynamic measures" (page 869, [50]).

One weakness of fMRI and PET activation paradigms is that the temporal resolution is (relatively) poor. Even with a rapid fMRI design, the acquisition sums data over 1-2 seconds, and there is a delay of 4-6 seconds between the relevant neuronal event, and the peak BOLD response. By contrast, MEG provides nearly simultaneous (with respect to the neuronal events) recording at $>10^{3} \mathrm{msec}$ temporal resolution. This means that subtle changes are more likely to be detected, and we have the opportunity for a more fine-grained analysis of functional connectivity (e.g., [51]). Alterations in signal characteristics may get lost in the noise of a 2 second acquisition of fMRI (i.e., TR = 2000), but may be easily detected with MEG. This high temporal resolution allows the measurement of the dynamics of the oscillatory activity, and as a consequence establishing the functional interaction between brain regions at specific frequency bands. Therefore, MEG provides a four-dimensional view of brain function (space time frequency connectivity) which offers a better description of the consequences of neurological diseases on the functional networks which support cognitive functions. MEG may have potential as a biomarker of $\mathrm{AD}$, and we must evaluate the relative merits of the methodology in neurodegenerative disease.

As noted above, EEG measures of brain function have shown that the integrity of brain oscillatory activity is a good index of its functional state. Indeed, over the last several years, there has been an increase in interest in what is known as the "default mode network" which was first identified by using positron emission tomography (PET) and functional MRI [52]. Although clinical MRI scanners are now capable of routinely gathering resting state measures of cerebral blood flow, there is usually no analysis of functional networks during the "resting" state, as this requires offline processing (e.g., [53]). This is unfortunate because the "default mode" appears to be altered in cortical dementias [51, 54, 55] and such information could be useful diagnostically. While default mode networks detected with MRI oscillate below $0.5 \mathrm{~Hz}$, resting state measures acquired in MEG can detect higher frequency oscillations. Dipole density or minimum current estimates have provided important information about cortical abnormalities that lead to the clinical progression of AD [56-58]. Although MEG is relatively less sensitive to subcortical abnormalities compared to cortical changes, it can assess function in $\sim 70 \%$ of the fissural cortex, (due to the alignment of the cortical columns relative to the MEG sensors). Thus, MEG has a high likelihood of detecting subtle alterations in cortical function [59].

MEG has already shown promise in terms of detecting $\mathrm{AD}$ in its earliest clinical stage (see $[60,61])$. One study of 15 $\mathrm{AD}$ patients localized the generators of focal magnetic slow waves during an eyes-open resting condition using a simple dipole model [62]. There was an increase in the number of dipoles in the delta and theta bands, and significant slowing in brain electrical activity in the temporal and parietal regions of both hemispheres of the $\mathrm{AD}$ patients [63]. Critically, the slow-wave activity in the right temporal parietal regions varied as a function of degree of cognitive impairment, whereas the activity in the left temporal areas was associated with functional status [62]. The alterations in brain function that are detected with MEG, especially those in the temporal regions, are significantly correlated with the relative volume of the lateral and medial temporal 
lobes $[64,65]$. This establishes a link between altered brain structure and altered brain function measured in two different modalities (i.e., MRI and MEG).

MEG may also be useful in aiding with some of the differential diagnosis that occurs in the context of AD. Specifically, the "profile" of neuromagnetic activity measured using the event-related magnetic response in a memory task, includes significantly reduced activity in the left temporal lobe in $\mathrm{AD}$ patients. By contrast, individuals with late onset depression, do not differ from nondemented cognitively normal elderly individuals $[66,67]$. Thus, there seems to be some degree of specificity of the neuromagnetic abnormalities observed in AD.

MEG shows particular promise in predicting the development of MCI from normal cognition. In a study of 15 healthy subjects, five of whom developed MCI two years later, those who showed cognitive decline had a lower number of activity sources (400-800 ms frequency range) in the left medial temporal lobe compared to the individuals who did not show cognitive decline [68]. Similarly, when individuals with MCI are studied after two years of followup, the relative risk of developing $\mathrm{AD}$ increased more than three times among those individuals who had significantly elevated number of dipole density scores in the delta frequency range [56]. Taken together these data demonstrate that MEG may not only be able to predict risk of developing AD from MCI, but may be able to detect brain functional abnormalities in cognitively normal individuals. Because MEG is noninvasive and does not involve the use of contrast agents or radiotracers, it can be easily repeated as often as necessary as a method for tracking disease progression.

Critical to understanding the relationships between brain function and cognition is the concept of functional connectivity. Brain function has been studied from the standpoint of functional segregation or specialization in an effort to localize cognitive functions in specific brain regions (see [69-71] for discussion). Modern views of brain organization, when coupled with more advanced statistical analysis techniques, have allowed us to study the relationship among brain regions and how they affect behavior [72], that is, the concept of functional integration studied with functional connectivity [73]. As such, brain networks represent complex systemic architecture, with a balance between segregation and integration of information. Functional connectivity refers to the statistical interdependence between neurophysiological data that is recorded simultaneously from a variety of different brain regions.

Since the early days of modern research in the pathology of $\mathrm{AD}$, there has been a discussion of concepts of disrupted connectivity among brain regions as being responsible for some of the earliest cognitive changes that occur in the disorder (e.g., [74]. This disruption of the anatomical/functional connections in $\mathrm{AD}$ led to the idea of a disconnection syndrome as being potentially responsible for much of the cognitive loss, at least early in the disease. This abnormality in functional connectivity suggests abnormal interactions between neural systems that typically interact to support cognition and behavior. Because the pathological changes associated with $\mathrm{AD}$ begin several decades before the onset of the clinical syndromes, it is important to evaluate whether the functional profiles are affected in the preclinical stages, including MCI, and even in the context of normal aging.

MEG provides data about three-dimensional space (space and time), as well as frequency bands, which allows us to address the question of which areas are functionally linked (i.e., connectivity), and at what point in the information processing stream the linkages occur. In MEG, the statistical correlation between two magnetic time series can be measured through linear and nonlinear methods including spectral coherence, phase synchronization, or generalized synchronization. Long-range synchronization between signals; that is, oscillatory activity, originating in relatively distant neuronal populations is one potential mechanism for communication and integration of information in the brain [75-77]. Thus, functional connectivity can be viewed as being closely related to the concept of synchrony which is the most common, economical, and biologically plausible mechanism for information communication in the brain.

In a recent series of papers examining visual working memory, Palva and colleagues have demonstrated how synchrony can support memory $[78,79]$. In their studies, they mapped the dynamics of network synchrony during the performance of a memory task and found that there was sustained phase synchrony in parietal/frontal circuits in three frequency bands. Perhaps most important was the finding that the synchrony increased as a function of the memory load. They suggested that the synchrony among the various brain regions could reflect a compensatory mechanism to help deal with retention of information, when that information load rises above working memory "capacity" (usually in the range of 3-4 items). This is a provocative finding in the present context because the data suggests that there is some form of compensatory response possible in the early stages of MCI and AD [73, 80, 81].

Synchronization Likelihood (SL) has been used to differentiate between individuals with $\mathrm{AD}$ to subjects with no cognitive impairment in MEG [39, 51, 82]. These studies show, in general, a loss of long-distance synchronization in $\mathrm{AD}$ patients. In $\mathrm{MCI}$, similar reductions in long-distance connections were also noted, even in a resting state. However during a memory task, patients with MCI showed increased SL values relative to healthy controls [83]. These latter data argue against $\mathrm{MCI}$ as a disconnection syndrome, but also must be viewed in the context that there is a functional compensatory response that occurs during the MCI phase [81].

The earliest clinical indicators of potential alteration in brain function can often be symptoms of "subjective memory complaints" that are not accompanied by alterations in neuropsychological test performance. Patients with subjective memory complaints showed higher MEG activation than control subjects in posterior ventral regions of the cortex, as well as in the parietal/occipital regions [84]. No statistically significant differences were found between patients with MCI and individuals with subjective memory complaints, indicating a certain degree of similarity between individuals with complaints of memory loss and those with documented alterations in cognitive functions. This suggests 
at a minimum that within the group of individuals with subjective memory complaints there are some who will progress to a stage of MCI and eventually AD [85]. Further, in the earliest preclinical stages of the dementia syndrome, MEG may be capable of detecting alterations in the functional organization of the central nervous system that indicate the existence of a degenerative process.

$\mathrm{AD}$ is considered a disease of the cholinergic transmitter system. This is due in part to the fact that one of the earliest manifestations of AD pathology is a loss of cholinergic neurons arising from the nucleus basalis of Meynert in the basal forebrain $[86,87]$ which can be visualized in structural brain imaging [88-92]. Indeed, atrophy of the basal forebrain is a marker for rapid decline from normal cognition to dementia within four years [93]. Indeed, challenging the cholinergic system by the administration of scopolamine to cognitively healthy elders results in greater decline in memory than in younger individuals [94]. Critical to the present discussion is the fact that MEG studies have found that spontaneous brain activity and cortical auditory processing [95] which are impaired in $\mathrm{AD}$, are themselves modulated by the scopolamine $[96,97]$. Because MEG appears to be sensitive enough to detect short-term changes in brain activity by cholinergic modulation it may be useful as a biomarker for the modulation of the cholinergic system, or of the neural networks responsible for spontaneous background activity during the course of a typical pharmacological trial.

As in any other medical procedure MEG needs the cooperation of the patient to perform an accurate scan. Just like MRI and PET, patient movement inside the scanner produces a distortion of the images, which reduces the utility of the results. For MEG imaging, resting state studies with MEG require only that the subject is seated (or lying) in a comfortable position, and remains motionless; MEG manufacturers have developed head tracking systems to correct for any inadvertent movements. During cognitive tasks, it is necessary to acquire a large number of trials so that the data can be analyzed on a single subject basis, so there is a necessary tradeoff between time in the scanner and data acquisition. The large number of trials (normally 120 in a memory task) allows for an analysis of the within-subject reliability of the data (i.e., comparing the first half and second half of the trials), something not generally possible with PET or fMRI studies.

Before the utility of MEG can be rigorously evaluated in $\mathrm{AD}$ and related dementias, there are some critical prerequisite data that need to be gathered, including determining the test-retest reliability, and the stability of the signal over time. That is, in order for MEG to be useful in tracking the natural history of CNS function, or to be used in evaluating pharmacotherapy (for example), we must first understand the extent to which the data are reproducible, both over the short term (i.e., reliability) and longer term (i.e., stability). Critically, high reliability and/or stability of the MEG response are necessary preconditions for MEG validity. Further, different centers must be able to identify the same biomagnetic signature in order for any putative biomarker to be useful. Finally, it is equally important to analyze the reliability and stability of the behavioral task (e.g., the modified Sternberg probe task used in many MEG AD studies) itself. It is critical that the memory task (or other behavioral probe) is not only reliable, but that any changes in performance over time are also reflected by changes in the larger battery of neuropsychological tests (i.e., concurrent validity).

To date, the bulk of clinically relevant MEG research uses activation protocols focusing either on somatosensory functions (e.g., [98]) or, more prominently, language tasks used in the context of presurgical functional localization studies [99]. A (relatively) early review of the field [100] found that MEG was generally reliable and valid (relative to sodium amytal studies), while source localization varies within a range of 1.5-3 $\mathrm{mm}$ [100] which is less than one voxel of MRI data. MEG signal reliability for a single region was rho $=.42$, and the median correlation across more than 20 regions was.61 (with only one region having a correlation less than .50) [101]. Thus, at least for language-based tasks, the ability to localize MEG signal appears good [99].

Once the reliability and stability of MEG are established for these tasks and patient groups, there must be a demonstration that a specific "signature" of $\mathrm{AD}$ has concurrent validity with other known biomarkers such as brain structural and cerebral metabolism (and amyloid imaging) (e.g., [65]). In addition, there should be predictive validity in that the presence of an abnormality identified by MEG should correlate with pathological changes in AD identified at autopsy.

Because MEG has only recently been applied to the study of neurodegenerative diseases, there are relatively few centers with the capability and interest in studying the potential utility of MEG. These centers are widely distributed around the world adding to the logistic complexity of completing a study of reliability and stability. However, if such a crosscenter study could be accomplished, this would result in increased generalizability of the findings as they would have been acquired on different machines in different countries using different at-risk populations.

Given this background, we recently formed an international collaborative group for the study of the utility of MEG for the preclinical detection of AD. This collaborative group has the potential to validate previous findings with MEG, and to demonstrate the suitability of including MEG in the study of the earliest diagnosis and detection of $\mathrm{AD}$ and related dementias. Three of the centers have extensive experience studying the capability of MEG in differentiating between healthy aging and $\mathrm{AD}$, and as a consequence of this research there are several principal findings that could potentially serve as signature biomarkers. These include (1) a delay in the latency of the N100 m signal which is an index of stimulus detection; (2) a delay in the mismatch negativity in auditory cortex as a measure of automatic stimulus detection and delay of memory trace; (3) an increase in parietal activity in the delta frequency band in posterior cortical regions; (4) a reduced number of biomagnetic sources in the posterior cortical regions during performance of memory tasks; (5) higher local SL value but lower long-distance SL values in spontaneous (i.e., resting) brain activity. 
As a first step in the process of determining the reliability and validity of MEG in the diagnosis of $\mathrm{AD}$, the collaborative group will determine the cross-center reliability of measuring biomagnetic signal using standardized techniques, on the same model equipment, from the same equipment manufacturer. The protocol includes both resting state (eyes open, eyes closed), a memory probe task and a mismatchnegativity task [102]. In addition, all of the subjects will undergo high-resolution MRI, including anatomical sequences (i.e., MP-RAGE) as well as those to identify white matter abnormalities (e.g., FLAIR, DTI). All of the data will be sent to a central analysis center (Madrid) where they will be processed (blind) and the results recombined by an independent data analyst in order to determine centerto-center reliability. Signal analysis will also be conducted independently in Helsinki. The data will then be analyzed in an attempt to replicate not only at a group level, but also a single subject level. Of particular interest is the extent to which it is possible to use classification algorithms based on comparing $\mathrm{AD}$ patients and control subjects in order to identify MCI in the context of healthy aging, something that will be spearheaded from Pittsburgh (e.g., [103]).

Having established the inter- and intracenter variability, we will have established a necessary precondition for studies of concurrent validity relative to the clinical diagnosis, and to standard measures of brain structural abnormalities (e.g., decreased volume of the hippocampus). Having established these initial standards, only then it will be possible to embark on large-scale multicenter trials to fully examine the relative merits of MEG in the diagnosis and early detection of neurodegenerative pathologies.

Because MEG is a relatively new neuroimaging technology and is used extensively only in centers with significant research operations, it is necessary to establish these criteria not only on a multisite basis, but also a multinational basis. No single center will have sufficient cases or the analytic wherewithal to complete the process in isolation. It is only by virtue of these multinational collaborative efforts that MEG can be fairly evaluated for potential use in $\mathrm{AD}$.

\section{Acknowledgments}

Preparation of this manuscript was supported in part by an unrestricted educational grant from the Elekta Corp. to E. Zamrini. In addition, the work was partially supported by funds from the National Institutes of Health (R03MH081721, P01AG05133) and the Fulbright Commission to J. T. Becker, and from the Spanish Ministry of Innovation and Science (PSI2009-14415-C03-01) to F. Maestu The Magnetoencephalography International Consortium of Alzheimer's Disease investigators and collaborators include University of Utah: E. Zamrini, M. Funke, M. Riley; Complutense University of Madrid and Technical University of Madrid: F. Maestu, R. Bajo, F. del Pozo, N. Castellanos, A. Fernandez; Helsinki Central Hospital and University of Helsinki: J. Makela, K. C. Stam, B. W. van Dijk, E. Pekkonen; Carnegie-Mellon University: G. Sudre; University of Pittsburgh: A. Bagic, J. T. Becker, M. Fabrizio, A. Haridis, and W. Fassbinder.

\section{References}

[1] G. McKhann, D. Drachman, M. Folstein, R. Katzman, D. L. Price, and E. Stadlan, "Clinical diagnosis of Alzheimer's disease: report of the NINCDS-ADRDA work group under the auspices of Department of Health and Human Services Task Force on Alzheimer's disease," Neurology, vol. 34, no. 7, pp. 939-944, 1984.

[2] APA, Diagnostic and Statistical Manual of Mental Disorders, APA, New York, NY, USA, 3rd edition, 1980.

[3] APA, Diagnostic and Statistical Manual on Mental DisordersRevised (DSM-III-R), American Psychiatric Press, Washington, DC, USA, 3rd edition, 1987.

[4] R. C. Petersen, G. E. Smith, S. C. Waring, R. J. Ivnik, E. Kokmen, and E. G. Tangelos, "Aging, memory, and mild cognitive impairment," International Psychogeriatrics, vol. 9, no. 1, pp. 65-69, 1997.

[5] R. C. Petersen, G. E. Smith, S. C. Waring, R. J. Ivnik, E. G. Tangalos, and E. Kokmen, "Mild cognitive impairment: clinical characterization and outcome," Archives of Neurology, vol. 56, no. 3, pp. 303-308, 1999.

[6] R. C. Petersen, R. Doody, A. Kurz et al., "Current concepts in mild cognitive impairment," Archives of Neurology, vol. 58, no. 12, pp. 1985-1992, 2001.

[7] R. Petersen, Mild Cognitive Impairment: Aging to Alzheimer's Disease, Oxford University Press, New York, NY, USA, 2003.

[8] R. C. Petersen, "Mild cognitive impairment as a diagnostic entity," Journal of Internal Medicine, vol. 256, no. 3, pp. 183194, 2004.

[9] R. C. Petersen, R. O. Roberts, D. S. Knopman et al., "Mild cognitive impairment: ten years later," Archives of Neurology, vol. 66, no. 12, pp. 1447-1455, 2009.

[10] J. C. Morris, "Mild cognitive impairment is early-stage Alzheimer disease: time to revise diagnostic criteria," Archives of Neurology, vol. 63, no. 1, pp. 15-16, 2006.

[11] J. C. Morris and J. Cummings, "Mild cognitive impairment (MCI) represents early-stage Alzheimer's disease," Journal of Alzheimer's Disease, vol. 7, no. 3, pp. 235-239, 2005.

[12] J. C. Morris, M. Storandt, J. P. Miller et al., "Mild cognitive impairment represents early-stage Alzheimer disease," Archives of Neurology, vol. 58, no. 3, pp. 397-405, 2001.

[13] J. C. Morris, D. W. McKeel, M. Storandt et al., "Very mild Alzheimer's disease: informant-based clinical, psychometric, and pathologic distinction from normal aging," Neurology, vol. 41, no. 4, pp. 469-478, 1991.

[14] Z. S. Khachaturian, "Diagnosis of Alzheimer's disease," Archives of Neurology, vol. 42, no. 11, pp. 1097-1105, 1985.

[15] M. Gearing, S. S. Mirra, J. C. Hedreen, S. M. Sumi, L. A. Hansen, and A. Heyman, "The consortium to establish a registry for Alzheimer's disease (CERAD). Part X. Neuropathology confirmation of the clinical diagnosis of Alzheimer's disease," Neurology, vol. 45, no. 3, pp. 461-466, 1995.

[16] S. S. Mirra, A. Heyman, D. McKeel et al., "The Consortium to Establish a Registry for Alzheimer's Disease (CERAD). Part II. Standardization of the neuropathologic assessment of Alzheimer's disease," Neurology, vol. 41, no. 4, pp. 479-486, 1991.

[17] B. Dubois, H. H. Feldman, C. Jacova et al., "Research criteria for the diagnosis of Alzheimer's disease: revising the NINCDS-ADRDA criteria," Lancet Neurology, vol. 6, no. 8, pp. 734-746, 2007.

[18] L. S. Schneider, "Organising the language of Alzheimer's disease in light of biomarkers," The Lancet Neurology, vol. 9, no. 11, pp. 1044-1045, 2010. 
[19] H. J. Aizenstein, R. D. Nebes, J. A. Saxton et al., "Frequent amyloid deposition without significant cognitive impairment among the elderly," Archives of Neurology, vol. 65, no. 11, pp. 1509-1517, 2008.

[20] D. A. Wolk, J. C. Price, J. A. Saxton et al., "Amyloid imaging in mild cognitive impairment subtypes," Annals of Neurology, vol. 65, no. 5, pp. 557-568, 2009.

[21] J. R. Cirrito, K. A. Yamada, M. B. Finn et al., "Synaptic activity regulates interstitial fluid amyloid- $\beta$ levels in vivo," Neuron, vol. 48, no. 6, pp. 913-922, 2005.

[22] H. Berger, "Uber das elektrenkephalogramm des menschen," Archiv fur Psychiatrie, vol. 98, no. 1, pp. 16-60, 1931.

[23] H. Berger, "Uber das elektrenkephalogramm des menschen," Archiv fur Psychiatrie, vol. 98, no. 1, pp. 231-254, 1932.

[24] C. Babiloni, G. Binetti, E. Cassetta et al., "Mapping distributed sources of cortical rhythms in mild Alzheimer's disease. A multicentric EEG study," NeuroImage, vol. 22, no. 1, pp. 57-67, 2004.

[25] T. Dierks, R. Ihl, L. Frolich, and K. Maurer, "Dementia of the Alzheimer type: effects on the spontaneous EEG described by dipole sources," Psychiatry Research, vol. 50, no. 3, pp. 151$162,1993$.

[26] R. P. Brenner, C. F. Reynolds, and R. F. Ulrich, "Diagnostic efficacy of computerized spectral versus visual EEG analysis in elderly normal, demented and depressed subjects," Electroencephalography and Clinical Neurophysiology, vol. 69, no. 2, pp. 110-117, 1988.

[27] J. Jeong, "EEG dynamics in patients with Alzheimer's disease," Clinical Neurophysiology, vol. 115, no. 7, pp. 14901505, 2004.

[28] L. L. Smits, M. Liedorp, T. Koene et al., "EEG abnormalities are associated with different cognitive profiles in Alzheimer's disease," Dementia and Geriatric Cognitive Disorders, vol. 31, no. 1, pp. 1-6, 2011.

[29] M. Grunwald, F. Busse, A. Hensel et al., "Theta-power differences in patients with mild cognitive impairment under rest condition and during haptic tasks," Alzheimer Disease and Associated Disorders, vol. 16, no. 1, pp. 40-48, 2002.

[30] V. Jelic, S. E. Johansson, O. Almkvist et al., "Quantitative electroencephalography in mild cognitive impairment: longitudinal changes and possible prediction of Alzheimer's disease," Neurobiology of Aging, vol. 21, no. 4, pp. 533-540, 2000.

[31] C. Huang, L. O. Wahlund, T. Dierks, P. Julin, B. Winblad, and V. Jelic, "Discrimination of Alzheimer's disease and mild cognitive impairment by equivalent EEG sources: a crosssectional and longitudinal study," Clinical Neurophysiology, vol. 111, no. 11, pp. 1961-1967, 2000.

[32] G. Rodriguez, F. Copello, P. Vitali, G. Perego, and F. Nobili, "EEG spectral profile to stage Alzheimer's disease," Clinical Neurophysiology, vol. 110, no. 10, pp. 1831-1837, 1999.

[33] V. Jelic, M. Shigeta, P. Jnlin, O. Almkvist, B. Winblad, and L. O. Wahhmd, "Quantitative electroencephalography power and coherence in Alzheimer's disease and mild cognitive impairment," Dementia, vol. 7, no. 6, pp. 314-323, 1996.

[34] C. Babiloni, R. Ferri, G. Binetti et al., "Directionality of EEG synchronization in Alzheimer's disease subjects," Neurobiology of Aging, vol. 30, no. 1, pp. 93-102, 2009.

[35] P. L. Nunez, R. B. Silberstein, Z. Shi et al., "EEG coherency II: experimental comparisons of multiple measures," Clinical Neurophysiology, vol. 110, no. 3, pp. 469-486, 1999.

[36] P. L. Nunez, R. Srinivasan, A. F. Westdorp et al., "EEG coherency I: statistics, reference electrode, volume conduction, Laplacians, cortical imaging, and interpretation at multiple scales," Electroencephalography and Clinical Neurophysiology, vol. 103, no. 5, pp. 499-515, 1997.

[37] D. V. Moretti, G. B. Frisoni, M. Pievani et al., "Cerebrovascular disease and hippocampal atrophy are differently linked to functional coupling of brain areas: an EEG coherence study in MCI subjects," Journal of Alzheimer's Disease, vol. 14, no. 3, pp. 285-299, 2008.

[38] C. J. Stam and B. W. Van Dijk, "Synchronization likelihood: an unbiased measure of generalized synchronization in multivariate data sets," Physica D, vol. 163, no. 3-4, pp. 236$251,2002$.

[39] C. J. Stam, Y. Van Der Made, Y. A. L. Pijnenburg, and PH. Scheltens, "EEG synchronization in mild cognitive impairment and Alzheimer's disease," Acta Neurologica Scandinavica, vol. 108, no. 2, pp. 90-96, 2003.

[40] Y. A. L. Pijnenburg, Y. Vd Made, A. M. Van Cappellen Van Walsum, D. L. Knol, PH. Scheltens, and C. J. Stam, "EEG synchronization likelihood in mild cognitive impairment and Alzheimer's disease during a working memory task," Clinical Neurophysiology, vol. 115, no. 6, pp. 1332-1339, 2004.

[41] P. M. Rossini, C. Del Percio, P. Pasqualetti et al., "Conversion from mild cognitive impairment to Alzheimer's disease is predicted by sources and coherence of brain electroencephalography rhythms," Neuroscience, vol. 143, no. 3, pp. 793-803, 2006.

[42] M. A. Kramer, F. L. Chang, M. E. Cohen, D. Hudson, and A. J. Szeri, "Synchronization measures of the scalp electroencephalogram can discriminate healthy from Alzheimer's subjects," International Journal of Neural Systems, vol. 17, no. 2, pp. 61-69, 2007.

[43] J. Dauwels, F. Vialatte, T. Musha, and A. Cichocki, "A comparative study of synchrony measures for the early diagnosis of Alzheimer's disease based on EEG," NeuroImage, vol. 49, no. 1, pp. 668-693, 2010.

[44] P. R. Hof, C. Bouras, J. Constantinidis, and J. H. Morrison, "Selective disconnection of specific visual association pathways in cases of Alzheimer's disease presenting with Balint's syndrome," Journal of Neuropathology and Experimental Neurology, vol. 49, no. 2, pp. 168-184, 1990.

[45] D. Cohen, "Magnetoencephalography: detection of the brain's electrical activity with a superconducting magnetometer," Science, vol. 175, no. 4022, pp. 664-666, 1972.

[46] J. S. George, C. J. Aine, J. C. Mosher et al., "Mapping function in the human brain with magnetoencephalography, anatomical magnetic resonance imaging, and functional magnetic resonance imaging," Journal of Clinical Neurophysiology, vol. 12, no. 5, pp. 406-431, 1995.

[47] D. S. Manoach, E. F. Halpern, T. S. Kramer et al., "Test-retest reliability of a functional MRI working memory paradigm in normal and schizophrenic subjects," American Journal of Psychiatry, vol. 158, no. 6, pp. 955-958, 2001.

[48] R. K. Heaton, T. D. Marcotte, M. Rivera Mindt et al., "The impact of HIV-associated neuropsychological impairment on everyday functioning," Journal of the International Neuropsychological Society, vol. 10, no. 3, pp. 317-331, 2004.

[49] S. Taulu and R. Hari, "Removal of magnetoencephalographic artifacts with temporal signal-space separation: demonstration with single-trial auditory-evoked responses," Human Brain Mapping, vol. 30, no. 5, pp. 1524-1534, 2009.

[50] A. C. Papanicolaou, P. G. Simos, E. M. Castillo et al., "Magnetocephalography: a noninvasive alternative to the Wada procedure," Journal of Neurosurgery, vol. 100, no. 5, pp. 867876, 2004. 
[51] C. J. Stam, B. F. Jones, I. Manshanden et al., "Magnetoencephalographic evaluation of resting-state functional connectivity in Alzheimer's disease," NeuroImage, vol. 32, no. 3, pp. 1335-1344, 2006.

[52] M. E. Raichle, A. M. MacLeod, A. Z. Snyder, W. J. Powers, D. A. Gusnard, and G. L. Shulman, "A default mode of brain function," Proceedings of the National Academy of Sciences of the United States of America, vol. 98, no. 2, pp. 676-682, 2001.

[53] F. Bai, Z. Zhang, H. Yu et al., "Default-mode network activity distinguishes amnestic type mild cognitive impairment from healthy aging: a combined structural and resting-state functional MRI study," Neuroscience Letters, vol. 438, no. 1, pp. 111-115, 2008.

[54] R. L. Buckner, A. Z. Snyder, B. J. Shannon et al., "Molecular, structural, and functional characterization of Alzheimer's disease: evidence for a relationship between default activity, amyloid, and memory," Journal of Neuroscience, vol. 25, no. 34, pp. 7709-7717, 2005.

[55] A. Fernández, R. Hornero, A. Mayo, J. Poza, F. Maestu, and T. Ortiz Alonso, "Quantitative magnetoencephalography of spontaneous brain activity in alzheimer disease: an exhaustive frequency analysis," Alzheimer Disease and Associated Disorders, vol. 20, no. 3, pp. 153-159, 2006.

[56] A. Fernández, A. Turrero, P. Zuluaga et al., "Magnetoencephalographic parietal $\delta$ dipole density in mild cognitive impairment: preliminary results of a method to estimate the risk of developing Alzheimer disease," Archives of Neurology, vol. 63, no. 3, pp. 427-430, 2006.

[57] A. Fernández, J. M. García-Segura, T. Ortiz et al., "Proton magnetic resonance spectroscopy and magnetoencephalographic estimation of delta dipole density: a combination of techniques that may contribute to the diagnosis of Alzheimer's disease," Dementia and Geriatric Cognitive Disorders, vol. 20, no. 2-3, pp. 169-177, 2005.

[58] D. Osipova, E. Pekkonen, and J. Ahveninen, "Enhanced magnetic auditory steady-state response in early Alzheimer's disease," Clinical Neurophysiology, vol. 117, no. 9, pp. 19901995, 2006.

[59] A. Hillebrand and G. R. Barnes, "A quantitative assessment of the sensitivity of whole-head MEG to activity in the adult human cortex," NeuroImage, vol. 16, no. 3, part 1, pp. 638650, 2002.

[60] C. J. Stam, A. M. Van Cappellen van Walsum, Y. A. L. Pijnenburg et al., "Generalized synchronization of MEG recordings in Alzheimer's disease: evidence for involvement of the gamma band," Journal of Clinical Neurophysiology, vol. 19, no. 6, pp. 562-574, 2002.

[61] C. J. Stam, B. F. Jones, I. Manshanden et al., "Magnetoencephalographic evaluation of resting-state functional connectivity in Alzheimer's disease," NeuroImage, vol. 32, no. 3, pp. 1335-1344, 2006.

[62] A. Fernández, F. Maestu, C. Amo et al., "Focal temporoparietal slow activity in Alzheimer's disease revealed by magnetoencephalography," Biological Psychiatry, vol. 52, no. 7, pp. 764-770, 2002.

[63] D. Osipova, K. Rantanen, J. Ahveninen et al., "Source estimation of spontaneous MEG oscillations in mild cognitive impairment," Neuroscience Letters, vol. 405, no. 1-2, pp. 5761, 2006.

[64] A. Fernández, J. Arrazola, F. Maestu et al., "Correlations of hippocampal atrophy and focal low-frequency magnetic activity in Alzheimer disease: volumetric MR imagingmagnetoencephalographic study," American Journal of Neuroradiology, vol. 24, no. 3, pp. 481-487, 2003.
[65] F. Maestu, J. Arrazola, A. Fernández et al., "Do cognitive patterns of brain magnetic activity correlate with hippocampal atrophy in Alzheimer's disease?" Journal of Neurology Neurosurgery and Psychiatry, vol. 74, no. 2, pp. 208-212, 2003.

[66] F. Maestu, A. Fernandez, P. G. Simos et al., "Profiles of brain magnetic activity during a memory task in patients with Alzheimer's disease and in non-demented elderly subjects, with or without depression," Journal of Neurology, Neurosurgery and Psychiatry, vol. 75, no. 8, pp. 1160-1162, 2004.

[67] A. Fernández, A. Rodriguez-Palancas, M. López-Ibor et al., "Increased occipital delta dipole density in major depressive disorder determined by magnetoencephalography," Journal of Psychiatry and Neuroscience, vol. 30, no. 1, pp. 17-23, 2005.

[68] F. Maestu, P. Campo, P. Gil-Gregorio, S. Fernández, A. Fernández, and T. Ortiz, "Medial temporal lobe neuromagnetic hypoactivation and risk for developing cognitive decline in elderly population: a 2-year follow-up study," Neurobiology of Aging, vol. 27, no. 1, pp. 32-37, 2006.

[69] K. J. Friston, C. D. Frith, P. F. Liddle, and R. S. J. Frackowiak, "Functional connectivity: the principal-component analysis of large (PET) data sets," Journal of Cerebral Blood Flow and Metabolism, vol. 13, no. 1, pp. 5-14, 1993.

[70] K. J. Friston, "Functional and effective connectivity in neuroimaging: a synthesis," Human Brain Mapping, vol. 2, no. 1-2, pp. 56-78, 1994.

[71] C. Buechel and K. J. Friston, "Characterising functional integration," in Human Brain Function, R. S. J. Frackowiak, K. J. Friston, C. D. Frith, R. J. Dolan, and J. C. Mazziotta, Eds., pp. 127-140, Scademic Press, San Diego, Calif, USA, 1997.

[72] A. R. McIntosh, C. L. Grady, L. G. Ungerleider, J. V. Haxby, S. I. Rapoport, and B. Horwitz, "Network analysis of cortical visual pathways mapped with PET," Journal of Neuroscience, vol. 14, no. 2, pp. 655-666, 1994.

[73] A. N. Herbster, T. Nichols, M. B. Wiseman, M. A. Mintun, S. T. Dekosky, and J. T. Becker, "Functional connectivity in auditory-verbal short-term memory in Alzheimer's disease," NeuroImage, vol. 4, no. 2, pp. 67-77, 1996.

[74] J. H. Morrison, J. Rogers, S. Scherr, R. Benoit, and F. E. Bloom, "Somatostatin immunoreactivity in neuritic plaques of Alzheimer's patients," Nature, vol. 314, no. 6006, pp. 9092, 1985.

[75] F. Varela, J. P. Lachaux, E. Rodriguez, and J. Martinerie, "The brainweb: phase synchronization and large-scale integration," Nature Reviews Neuroscience, vol. 2, no. 4, pp. 229239, 2001.

[76] P. Fries, "A mechanism for cognitive dynamics: neuronal communication through neuronal coherence," Trends in Cognitive Sciences, vol. 9, no. 10, pp. 474-480, 2005.

[77] A. K. Engel, P. Fries, and W. Singer, "Dynamic predictions: oscillations and synchrony in top-down processing," Nature Reviews Neuroscience, vol. 2, no. 10, pp. 704-716, 2001.

[78] J. M. Palva, S. Monto, S. Kulashekhar, and S. Palva, "Neuronal synchrony reveals working memory networks and predicts individual memory capacity," Proceedings of the National Academy of Sciences of the United States of America, vol. 107, no. 16, pp. 7580-7585, 2010.

[79] S. Palva, S. Monto, and J. M. Palva, "Graph properties of synchronized cortical networks during visual working memory maintenance," NeuroImage, vol. 49, no. 4, pp. 32573268, 2010.

[80] W. Dai, O. L. Lopez, O. T. Carmichael, J. T. Becker, L. H. Kuller, and H. M. Gach, "Abnormal regional cerebral blood 
flow in cognitively normal elderly subjects with hypertension," Stroke, vol. 39, no. 2, pp. 349-354, 2008.

[81] W. Dai, O. L. Lopez, O. T. Carmichael, J. T. Becker, L. H. Kuller, and H. M. Gach, "Mild cognitive impairment and alzheimer disease: patterns of altered cerebral blood flow at MR imaging," Radiology, vol. 250, no. 3, pp. 856-866, 2009.

[82] C. J. Stam, "Use of magnetoencephalography (MEG) to study functional brain networks in neurodegenerative disorders," Journal of the Neurological Sciences, vol. 289, no. 1-2, pp. 128$134,2010$.

[83] R. Bajo, F. Maestú, A. Nevado et al., "Functional connectivity in mild cognitive impairment during a memory task: implications for the disconnection hypothesis," Journal of Alzheimer's Disease, vol. 22, no. 1, pp. 183-193, 2010.

[84] F. Maestu, E. Baykova, J. M. Ruiz et al., "Increased biomagnetic activity in healthy elderly with subjective memory complaints," Clinical Neurophysiology, vol. 122, no. 3, pp. 499-505, 2011.

[85] I. Hernandez, P. Martinez-Lage, M. Alegret et al., "Subjective memory complaints are associated with impending cognitive decline," Neurology, vol. 74, supplement 2, p. A21, 2010.

[86] E. K. Perry, "The cholinergic system in old age and Alzheimer's disease," Age and Ageing, vol. 9, no. 1, pp. 1-8, 1980.

[87] P. J. Whitehouse, D. L. Price, and A. W. Clark, "Alzheimer disease: evidence for selective loss of cholinergic neurons in the nucleus basalis," Annals of Neurology, vol. 10, no. 2, pp. 122-126, 1981.

[88] G. B. Karas, P. Scheltens, S. A. R. B. Rombouts et al., "Global and local gray matter loss in mild cognitive impairment and Alzheimer's disease," NeuroImage, vol. 23, no. 2, pp. 708-716, 2004.

[89] G. Chételat, B. Desgranges, V. De la Sayette, F. Viader, F. Eustache, and J. C. Baron, "Mapping gray matter loss with voxel-based morphometry in mild cognitive impairment," NeuroReport, vol. 13, no. 15, pp. 1939-1943, 2002.

[90] S. J. Teipel, W. H. Flatz, H. Heinsen et al., "Measurement of basal forebrain atrophy in Alzheimer's disease using MRI," Brain, vol. 128, no. 11, pp. 2626-2644, 2005.

[91] J. C. Baron, G. Chételat, B. Desgranges et al., "In vivo mapping of gray matter loss with voxel-based morphometry in mild Alzheimer's disease," NeuroImage, vol. 14, no. 2, pp. 298-309, 2001.

[92] M. Grothe, L. Zaborszky, M. Atienza et al., "Reduction of basal forebrain cholinergic system parallels cognitive impairment in patients at high risk of developing alzheimer's disease," Cerebral Cortex, vol. 20, no. 7, pp. 1685-1695, 2010.

[93] A. M. Hall, R. Y. Moore, O. L. Lopez, L. Kuller, and J. T. Becker, "Basal forebrain atrophy is a presymptomatic marker for Alzheimer's disease," Alzheimer's and Dementia, vol. 4, no. 4, pp. 271-279, 2008.

[94] P. N. Tariot, S. V. Patel, C. Cox, and R. E. Henderson, "Agerelated decline in central cholinergic function demonstrated with scopolamine," Psychopharmacology, vol. 125, no. 1, pp. 50-56, 1996.

[95] E. Pekkonen, I. P. Jaaskelainen, M. Hietanen et al., "Impaired preconscious auditory processing and cognitive functions in Alzheimer's disease," Clinical Neurophysiology, vol. 110, no. 11, pp. 1942-1947, 1999.

[96] D. Osipova, J. Ahveninen, S. Kaakkola, I. P. Jaaskelainen, J. Huttunen, and E. Pekkonen, "Effects of scopolamine on MEG spectral power and coherence in elderly subjects," Clinical Neurophysiology, vol. 114, no. 10, pp. 1902-1907, 2003.
[97] E. Pekkonen, I. P. Jaaskelainen, S. Kaakkola, and J. Ahveninen, "Cholinergic modulation of preattentive auditory processing in aging," NeuroImage, vol. 27, no. 2, pp. 387-392, 2005.

[98] D. Lee, S. M. Sawrie, P. G. Simos, J. Killen, and R. C. Knowlton, "Reliability of language mapping with magnetic source imaging in epilepsy surgery candidates," Epilepsy and Behavior, vol. 8, no. 4, pp. 742-749, 2006.

[99] P. Missonnier, G. Gold, L. Fazio-Costa et al., "Early eventrelated potential changes during working memory activation predictrapid decline in mild cognitive impairment," The Journals of Gerontology, vol. 60A, no. 5, pp. 660-666, 2006.

[100] M. Liljeström, J. Kujala, O. Jensen, and R. Salmelin, "Neuromagnetic localization of rhythmic activity in the human brain: a comparison of three methods," NeuroImage, vol. 25, no. 3, pp. 734-745, 2005.

[101] S. S. Yoo, X. Wei, C. C. Dickey, C. R. G. Guttmann, and L. P. Panych, "Long-term reproducibility analysis of fMRI using hand motor task," International Journal of Neuroscience, vol. 115, no. 1, pp. 55-77, 2005.

[102] E. Pekkonen, "Mismatch negativity in aging and in Alzheimer's and Parkinson's disease," Audiology and NeuroOtology, vol. 5, no. 3-4, pp. 216-224, 2000.

[103] T. M. Mitchell, R. Hutchinson, R. S. Niculescu et al., "Learning to decode cognitive states from brain images," Machine Learning, vol. 57, no. 1-2, pp. 145-175, 2004. 


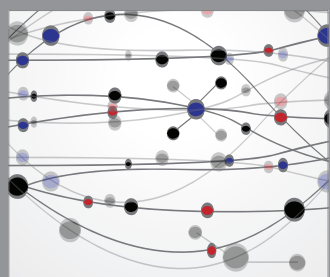

The Scientific World Journal
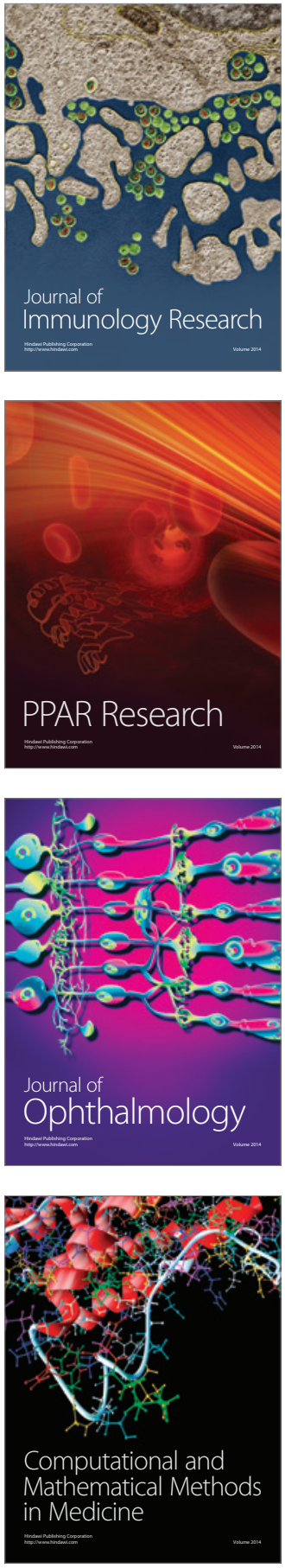

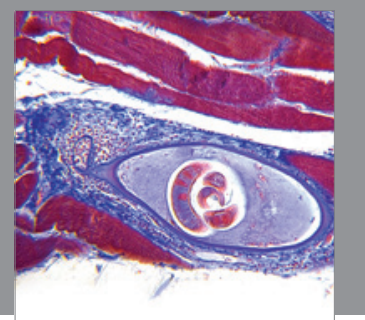

Gastroenterology

Research and Practice
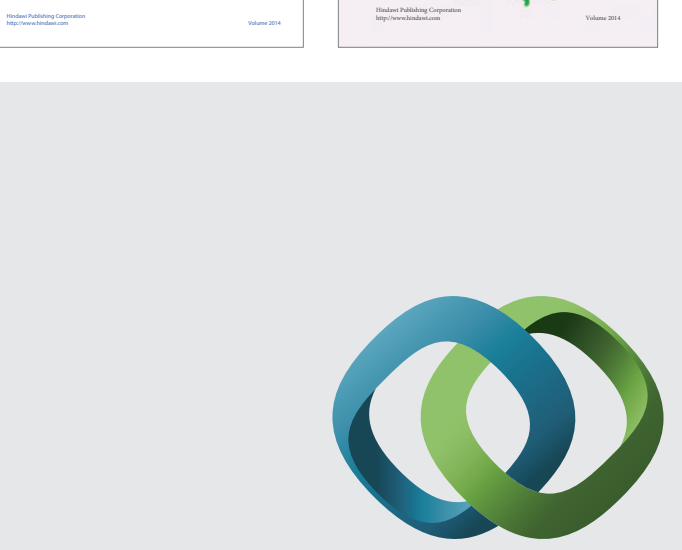

\section{Hindawi}

Submit your manuscripts at

http://www.hindawi.com
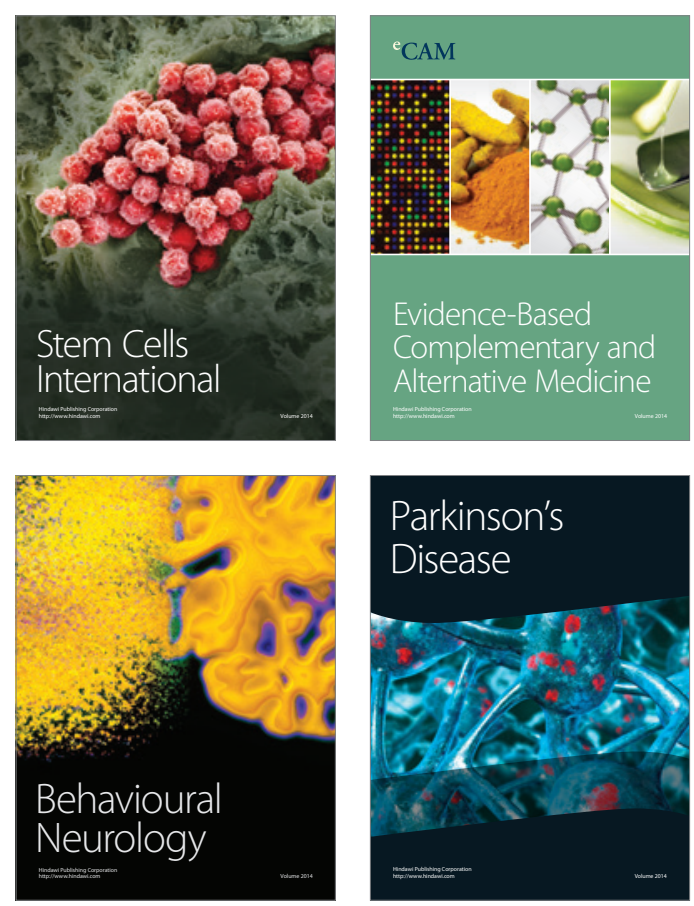

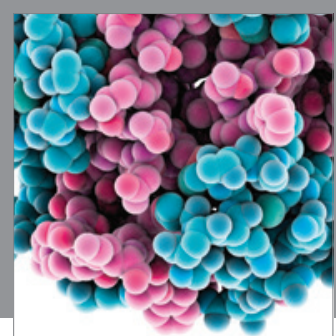

Journal of
Diabetes Research

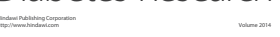

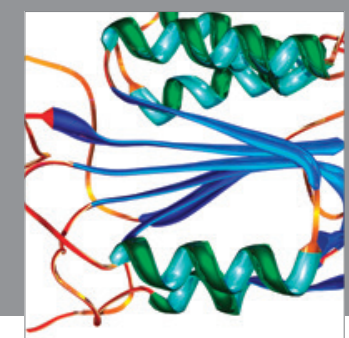

Disease Markers
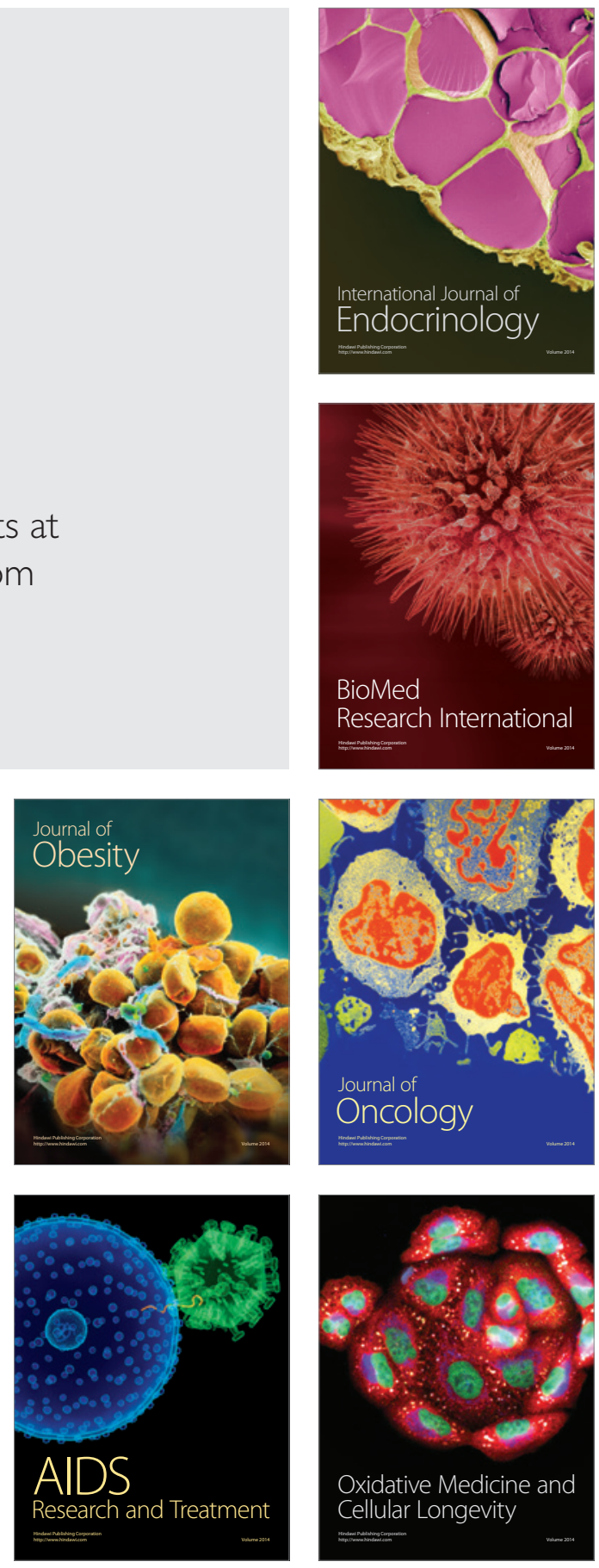